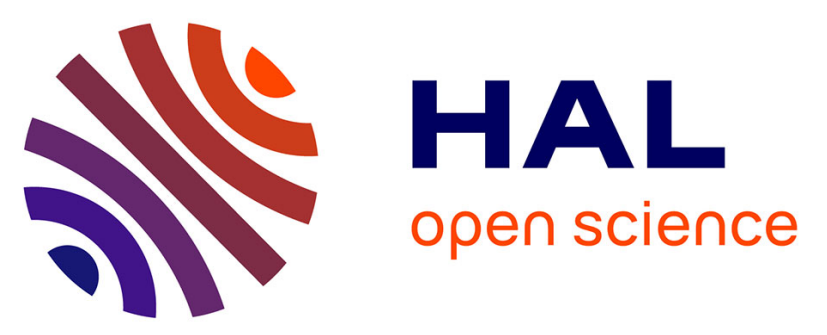

\title{
Evidence of L1(0) chemical order in CoPt nanoclusters: Direct observation and magnetic signature
}

Florent Tournus, Alexandre Tamion, Nils Blanc, Abdelkrim Hannour, Laurent Bardotti, Brigitte Prevel, Philippe Ohresser, Edgar Bonet, Thierry Epicier, Veronique Dupuis

\section{To cite this version:}

Florent Tournus, Alexandre Tamion, Nils Blanc, Abdelkrim Hannour, Laurent Bardotti, et al.. Evidence of L1(0) chemical order in CoPt nanoclusters: Direct observation and magnetic signature. Physical Review B: Condensed Matter and Materials Physics (1998-2015), 2008, 77 (14), pp.144411. 10.1103/PhysRevB.77.144411 . hal-01822684

\section{HAL Id: hal-01822684 https://hal.science/hal-01822684}

Submitted on 21 Jan 2022

HAL is a multi-disciplinary open access archive for the deposit and dissemination of scientific research documents, whether they are published or not. The documents may come from teaching and research institutions in France or abroad, or from public or private research centers.
L'archive ouverte pluridisciplinaire HAL, est destinée au dépôt et à la diffusion de documents scientifiques de niveau recherche, publiés ou non, émanant des établissements d'enseignement et de recherche français ou étrangers, des laboratoires publics ou privés. 


\title{
乌̧ \\ Evidence of $L 1_{0}$ chemical order in CoPt nanoclusters: Direct observation and magnetic signature
}

\author{
Florent Tournus, ${ }^{1, *}$ Alexandre Tamion, ${ }^{1}$ Nils Blanc, ${ }^{1}$ Abdelkrim Hannour, ${ }^{1}$ Laurent Bardotti, ${ }^{1}$ Brigitte Prével, ${ }^{1}$ \\ Philippe Ohresser, ${ }^{2}$ Edgar Bonet, ${ }^{3}$ Thierry Epicier ${ }^{4}$ and Véronique Dupuis ${ }^{1}$ \\ ${ }^{1}$ Université de Lyon, F-69000, France \\ and Université Lyon 1, Laboratoire PMCN, CNRS, UMR 5586, F-69622 Villeurbanne Cedex, France \\ ${ }^{2}$ Synchrotron SOLEIL, BP 48, 91192 Gif-sur-Yvette, France \\ ${ }^{3}$ CNRS/UJF, Institut Néel, BP 166, 38042 Grenoble Cedex 9, France \\ ${ }^{4}$ MATEIS, UMR CNRS 5510, INSA-Lyon, Université de Lyon, F-69621 Villeurbanne Cedex, France \\ (Received 11 January 2008; revised manuscript received 21 February 2008; published 10 April 2008)
}

\begin{abstract}
We report the synthesis and characterization of well-defined CoPt clusters with a mean diameter of $3 \mathrm{~nm}$, produced in ultrahigh vacuum conditions following a physical route. Samples made of diluted layers of $\mathrm{CoPt}$ clusters embedded in amorphous carbon have been studied by transmission electron microscopy. Highresolution observations have revealed the appearance of $L 1_{0}$ chemical order upon annealing, even for clusters with a $2 \mathrm{~nm}$ diameter, without cluster coalescence. The magnetic properties of both chemically disordered and ordered CoPt clusters embedded in amorphous carbon have then been measured by x-ray magnetic circular dichroism and superconducting quantum interference device magnetometry. Despite a striking change of the Co magnetic moment, the magnetic anisotropy of chemically ordered nanoparticles increases, with respect to the chemically disordered $A 1$ phase, in much lower proportions than what is observed for the bulk.
\end{abstract}

DOI: 10.1103/PhysRevB.77.144411

PACS number(s): 75.75.+a, 61.46.Df, 75.30.Gw, 68.37.Og

\section{INTRODUCTION}

Magnetic particles of a few nanometers diameter have attracted much attention both for their fundamental interest (the magnetic properties of low-coordinated atoms can display dramatic changes ${ }^{1,2}$ ) and for their potential applications. In particular, small permanent magnets at the nanoscale would make possible ultrahigh density magnetic storage devices. ${ }^{3}$ However, at such a size, the magnetization direction of a particle usually fluctuates at room temperature. Consequently, each ferromagnetic particle is in fact equivalent to a paramagnetic moment, with a vanishing average remanent magnetization. This phenomenon, which must be overcome for practical applications, is called superparamagnetism. In order to increase the thermal stability (or equivalently, to increase the magnetization relaxation time), great efforts have been directed toward an enhancement of the nanoparticles magnetic anisotropy. One of the preferred routes is to use magnetic alloys.

In this context, there have been considerable progresses in the synthesis procedures of FePt (Refs. 4-15) or CoPt (Refs. 16-31) nanoparticles. These alloys can all crystallize in the chemically ordered $L 1_{0}$ phase, corresponding to very high magnetocrystalline anisotropy for the bulk. ${ }^{32,33}$ Among these materials, we have investigated the specific case of CoPt. Unfortunately, despite significant advances, it is still very difficult to produce well-defined nanoparticles in the $L 1_{0}$ phase. Although this phase is the stable one at room temperature, an additional difficulty may come from the lowering of the order-disorder transition temperature (from the $L 1_{0}$ phase to the chemically disordered $A 1$ phase) due to the size reduction of the particles: ${ }^{34-42}$ Chemical order in small particles could then be thermodynamically unstable at room temperature. Moreover, chemical ordering is in general obtained by annealing, which goes with problems of pollution or coalescence, difficult to avoid. ${ }^{20,43-46}$
As a consequence, in spite of a huge number of results presented on these systems, there exists, to our knowledge, no convincing experimental study reporting a value of the intrinsic magnetic anisotropy for well-characterized $L 1_{0}$ CoPt nanoparticles. ${ }^{47}$ Indeed, it is a tricky task to ensure that CoPt nanoparticles have a well-known magnetic size, are chemically ordered, are noninteracting, etc., to correctly deduce the intrinsic properties of well-defined nanoparticles from macroscopic magnetic measurements. As a matter of fact, we may never be able to reach a magnetic anisotropy as high as the bulk one, for CoPt particles of a few nanometers diameter. This would constitute a serious setback for the expected technological applications.

In this paper, we report the synthesis and characterization of well-defined CoPt clusters with a mean diameter of $\simeq 3 \mathrm{~nm}$. After a short description of our original synthesis technique, following a physical route, and of the samples studied, we present transmission electron microscopy (TEM) observations revealing the appearance of the $L 1_{0}$ phase upon annealing, without particle coalescence. Then we show the results of magnetic measurements, by x-ray magnetic circular dichroism (XMCD) and superconducting quantum interference device (SQUID) magnetometry, on assemblies of both chemically disordered and ordered CoPt clusters embedded in amorphous carbon. We show that, despite a striking change of the Co magnetic moment, the magnetic anisotropy of chemically ordered nanoparticles increases in much lower proportions than what is observed for the bulk, with respect to the chemically disordered phase.

\section{SAMPLE PREPARATION}

CoPt nanoparticles are synthesized using a physical technique, as opposed to chemical synthesis of colloidal particles. We use the low energy cluster beam deposition 
a)

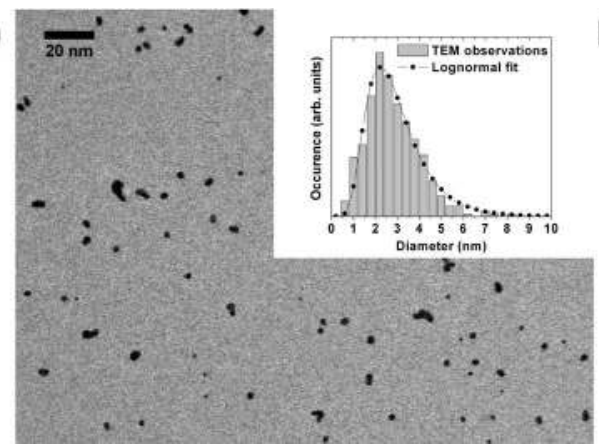

b)

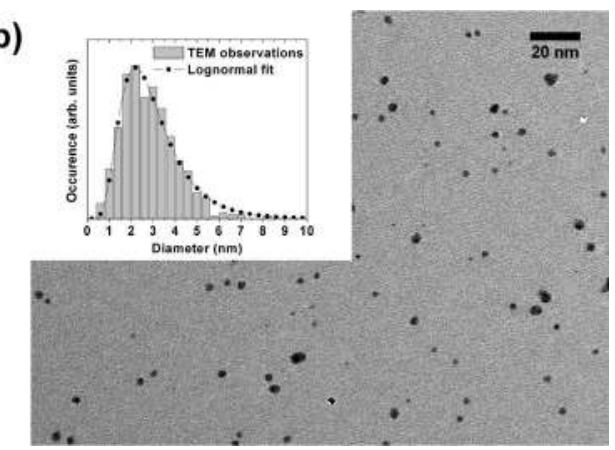

FIG. 1. TEM images of a 2D film of CoPt clusters (equivalent thickness $\simeq 0.5 \AA$ ) embedded in amorphous carbon, as prepared (a) and after annealing (b). The size histograms deduced by TEM images analysis, together with the best fits corresponding to a log-normal size distribution, are shown in the inset. The fit parameters [see Eq. (1)] are $d_{m}=2.58 \mathrm{~nm}, w=0.42$ and $d_{m}=2.67 \mathrm{~nm}, w=0.47$, respectively, for as-prepared and annealed clusters.

(LECBD) technique ${ }^{48,49}$ that allows us to grow thin films of nanoparticles deposited on a substrate. We will give a short description of this original technique.

Clusters are produced in a laser vaporization-gas condensation source similar to the one developed by Dietz et al., Milani and deHeer. ${ }^{50,51}$ Briefly, a plasma created by the impact of a Nd:YAG (yttrium aluminum garnet) laser beam focused on a rod is thermalized by injection of a continuous flow of helium at low pressure (typically 30 mbar) inducing the clusters growth. Clusters are subsequently stabilized and cooled down in the supersonic expansion taking place at the exit nozzle of the source. A low-energy cluster beam is then obtained, with clusters of different sizes, mostly neutral but also ionized (typically 1\% of ions), allowing the growth of thin cluster films on a substrate.

The main key points of this technique are the following. The samples are produced under ultrahigh vacuum conditions (base static pressure of $10^{-10} \mathrm{mbar}$, and around $10^{-8}$ mbar during deposition, due to the use of helium as carrier gas). This means that, contrary to other techniques, in particular the chemical synthesis involving ligands, the clusters surface is not altered. Moreover, as mentioned further, the samples can be protected by a capping layer in order to avoid any pollution or oxidation when they are exposed to air for subsequent characterizations. It is important to note that the clusters composition is determined by the one of the target used for laser vaporization. This means that the particles stoichiometry can easily be adjusted by simply changing the target. In the present case, we use an equiatomic $\mathrm{CoPt}$ rod, leading to nanoparticles with a composition very close to $\mathrm{Co}_{0.5} \mathrm{Pt}_{0.5}$. Indeed, Rutherford backscattering spectrometry (RBS) experiments ${ }^{52}$ indicate that the mean stoichiometry of the clusters films is $\mathrm{Co}_{0.56} \mathrm{Pt}_{0.44}$. Energy dispersive $\mathrm{x}$-ray spectroscopy (EDX) measurements on individual particles are in agreement with this result and show, within the precision of this technique, that the composition dispersion is quite low (around 10\%).

Another important feature of the LECBD technique is that the clusters arrive on the substrate in a soft-landing regime, following a random deposition scheme. Consequently, as long as there is no significant diffusion on the substrate, which is the case in this study, the interparticle distance is entirely controlled by the clusters density: The surface den- sity for two-dimensional (2D) samples or the volume density for three-dimensional samples where the clusters are embedded in a matrix. This allows us to adjust the strength of interparticle interactions and, by synthesizing highly diluted samples, to obtain thin films of clusters behaving as nearly isolated nanoparticles. Oppositely, there is, to our knowledge, no reported study on samples obtained from colloidal $\mathrm{CoPt}$ nanoparticles solutions where interparticle interactions are negligible. Moreover, the LECBD technique is highly versatile since clusters can be deposited on a large variety of substrates. For this study, we have used copper grids coated by a thin amorphous-carbon (a-C) layer, for TEM observations, and silicon substrates for magnetic measurements.

We have produced different samples of CoPt nanoparticles: 2D samples of CoPt clusters on a-C; 2D samples of $\mathrm{CoPt}$ clusters embedded in a-C, using an a-C capping layer; "sandwich" samples with two layers of CoPt particles embedded in a-C; and "mille-feuille" samples, on a silicon substrate, made of a large number of CoPt clusters layers (28 successive $2 \mathrm{D}$ cluster depositions) separated by a-C layers (a few nanometers thick). These two identical samples, used for magnetic characterizations, consist of diluted CoPt cluster layers, each one with an equivalent thickness of about $0.5 \AA$, corresponding to a cluster density around 2500 clusters per $\mu \mathrm{m}^{2}$ and a mean interparticle distance of $10 \mathrm{~nm}$. On the other hand, samples for TEM observations have been produced with various concentrations (i.e., cluster surface densities).

The size distribution of the deposited clusters is relatively sharp and corresponds to a log-normal curve. It slightly varies with the experimental conditions and depends on the material constituting the clusters. In the present case of $\mathrm{CoPt}$ nanoparticles, the clusters size, as deduced from size histograms measured by TEM (see Fig. 1), is well described by a log-normal function,

$$
f(d)=\frac{1}{w \sqrt{2 \pi}} \frac{1}{d} \exp \left[-\frac{1}{2}\left(\frac{\ln \left(d / d_{m}\right)}{w}\right)^{2}\right]
$$

with a median diameter $d_{m}=2.6 \pm 0.1 \mathrm{~nm}$ and a dispersion $w=0.42 \pm 0.05$ (corresponding to a mean diameter $\bar{d}$ $\simeq 2.9 \mathrm{~nm}$ and a standard deviation $\sigma \simeq 1.2 \mathrm{~nm}$ ). 

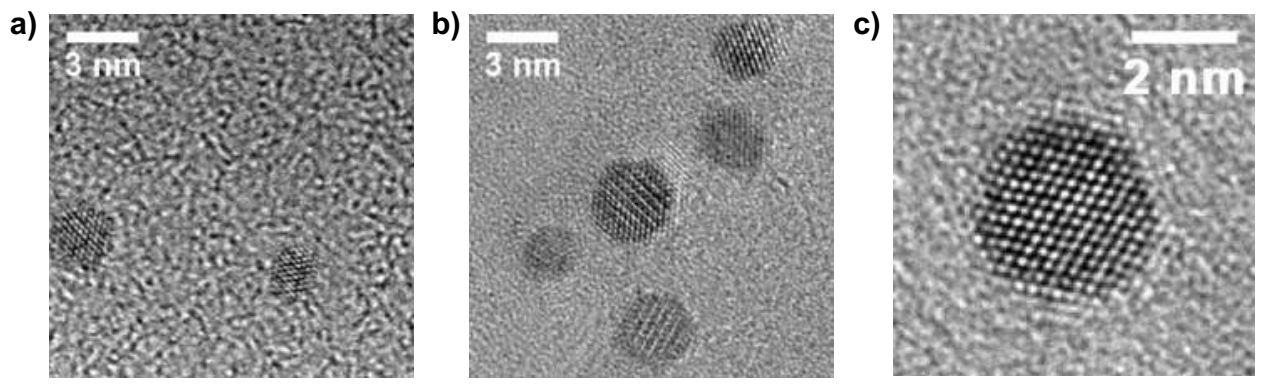

FIG. 2. HRTEM images of CoPt clusters of 2D films on amorphous carbon. (a) As-prepared clusters in the chemically disordered fcc A1 phase. (b,c) Annealed clusters: Some of them are in an orientation where $L 1_{0}$ chemical order is visible, whereas some others appear as chemically disordered crystals (c).

\section{TRANSMISSION ELECTRON MICROSCOPY OBSERVATIONS}

There is a double advantage of using a matrix, in our case $\mathrm{a}-\mathrm{C}$, in which the clusters are embedded. Indeed, on the one hand, it acts as a spacer, preventing clusters to come into contact both during the thin film growth and during an eventual post-deposition annealing (as discussed later); and on the other hand, it protects the clusters from pollution and oxidation, allowing us to expose the samples to air without any alteration of the CoPt particles properties.

Keeping this in mind, the choice of a-C as embedding matrix appears to be sound. Carbon indeed does not form any stable carbide with $\mathrm{Co}$ and $\mathrm{Pt}$, nor is it appreciably soluble in these elements, it is an efficient protection against oxidation, and most of all, it is transparent enough to electrons so that direct TEM observations are possible. We emphasize that, whereas other materials can represent interesting matrices (normal metals, or superconducting metals for particular applications ${ }^{53}$ ), a-C is really attractive because it is a light element and it is amorphous (contrary to nanocrystalline metallic or oxide matrices ${ }^{26}$ ): Having a direct observation of embedded clusters is of great help to ensure that the samples are made of well-separated nanoparticles of wellknown size, and that these characteristics are preserved upon annealing.

Following the widely used procedure, various CoPt clusters samples were annealed (2D films of bare clusters, 2D films coated by a-C, and "sandwich" samples) in order to promote $L 1_{0}$ chemical order in nanoparticles. Two hours annealing periods were performed at a $650{ }^{\circ} \mathrm{C}$ temperature and under high vacuum (pressure lower than $10^{-7} \mathrm{mbar}$ ), with or without a preliminary air exposure.

As it can be seen in Fig. 1, there is almost no clusters coalescence upon annealing when clusters are embedded in a-C: The size distribution is nearly the same, as well as the cluster density. There is, however, a noticeable change in the clusters shape: The circularity of the clusters projected surface increases with annealing, meaning that the nanoparticles are becoming more spherical. In addition, high resolution TEM (HRTEM) images (see Fig. 2) show that both asprepared and annealed clusters are well crystallized and display the typical $\{100\}$ and $\{111\}$ facets of a truncated octahedron.

Due to the smallness of inter-reticular distances change between the chemically disordered $A 1$ and the ordered $L 1_{0}$ phase, ${ }^{32}$ a distance measurement cannot be used to determine if the particles are in the $L 1_{0}$ phase. Moreover, there is no assurance that at such small sizes the interatomic distances are the same, or evolves in the same way, as in the bulk material. A similar cautiousness prevents us from using a tetragonalization measurement (the $c / a$ ratio, as determined for instance from $d_{111}$ and $d_{200}$ distances) to quantify the nanoparticles chemical order.

The best criteria to ensure that particles are not chemically disordered is to be able to observe a contrast which is not visible for face-centered-cubic (fcc) crystals [(001) or (110) for instance]. Indeed, the $L 1_{0}$ structure corresponds to successive pure $\mathrm{Co}$ and pure $\mathrm{Pt}$ atomic planes in the (001) direction. However, the particles must be in some particular orientations to allow such a contrast to manifest itself. This means that, even if the clusters are chemically ordered, only a small fraction will display a contrast corresponding to the Co-Pt alternation, because they are randomly oriented on the substrate. We would like to stress that, besides the statistical difficulty of finding correctly oriented particles, obtaining good quality HRTEM images of clusters with a diameter of only a few nanometers is highly challenging: Particles can move under the electron beam, they can even transform (Co evaporation for instance, as it is observed with nanobeam EDX analysis); the a-C layer can deform itself or even break down; a pollution can appear very quickly, and sometimes we observe the formation of an oxide shell around the clusters.

For annealed samples, we have been able to successfully observe nanoparticles in different orientations revealing a $L 1_{0}$-type contrast [not only a (001) contrast but also a (110) contrast]. For some particular particles, such as the one displayed in Fig. 3, which shows a remarkable (001) contrast, we have performed HRTEM images simulations. We have used the multislice method and the dynamical scattering theory, as implemented in the JEMS software. ${ }^{54,55}$ Simulations are in remarkable agreement with the experiment when considering a perfectly $L 1_{0}$-ordered cluster of the same size and orientation as the one observed by HRTEM (see Fig. 3). We can then positively conclude that such a contrast is not an artifact: It is a direct signature of the chemical order inside CoPt clusters after annealing. ${ }^{56}$ We also emphasize that the observation of a $L 1_{0}$-type contrast for annealed samples is not marginal at all: Although it is difficult to observe particles with an orientation revealing two different crystallographic planes, it is quite common to observe a (001) con- 
a)

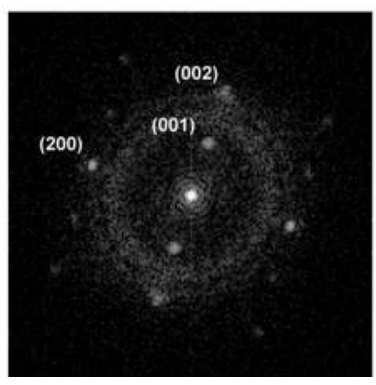

b)

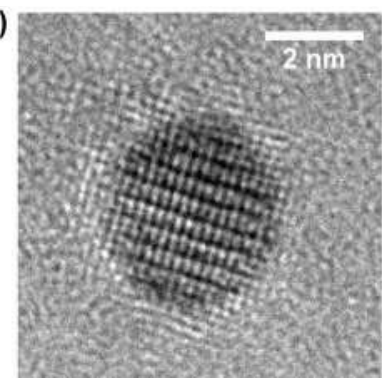

c)

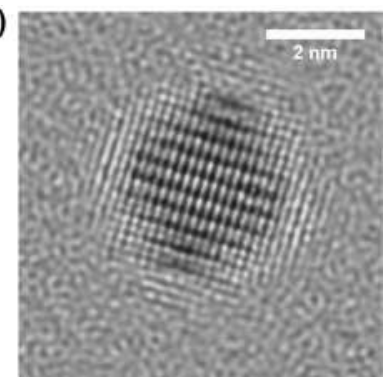

FIG. 3. (a) Fourier transform corresponding to the HRTEM image of the annealed CoPt cluster shown in (b). The (001) peak is the signature of $L 1_{0}$ chemical order corresponding to the strong contrast modulation visible in (b). (c) HRTEM image simulation obtained with a perfectly $L 1_{0}$ chemically ordered CoPt cluster, observed along the [010] direction, with a slight tilt.

trast alone. Nevertheless, since we can hardly evaluate the proportion of clusters displaying a $L 1_{0}$ contrast, the question of an incomplete chemical ordering in the sample will be addressed in Sec. V. At the opposite, for as-prepared samples, we have not been able to observe any $L 1_{0}$ contrast in HRTEM images. It shows that annealing is needed to promote chemical order in the nanoparticles, and that they are being produced in the chemically disordered fcc $A 1$ phase.

The observation of a $L 1_{0}$ contrast on HRTEM images constitutes direct evidence that individual particles are chemically ordered. HRTEM then appears as a powerful technique to investigate the $A 1 \rightarrow L 1_{0}$ transformation in nanoparticles. Other methods like $\mathrm{x}$-ray or electron diffraction may not be suited for samples made of diluted 2D assemblies of CoPt clusters with a diameter of $3 \mathrm{~nm}$ or less. Indeed, we have also succeeded in observing a (001) contrast in clusters of a $2 \mathrm{~nm}$ diameter (see Fig. 4). To our knowledge, chemical order had never been observed in so small CoPt nanoparticles. This result must be taken into account when examining thermodynamic predictions obtained from theoretical models. ${ }^{57}$ Note that the observation of these small $L 1_{0}$ clusters implies that they are thermodynamically stable, not only at room temperature, but at higher temperature since annealing has turned room temperature $A 1$ clusters into room temperature $L 1_{0}$ clusters. This pushes down the limit, and
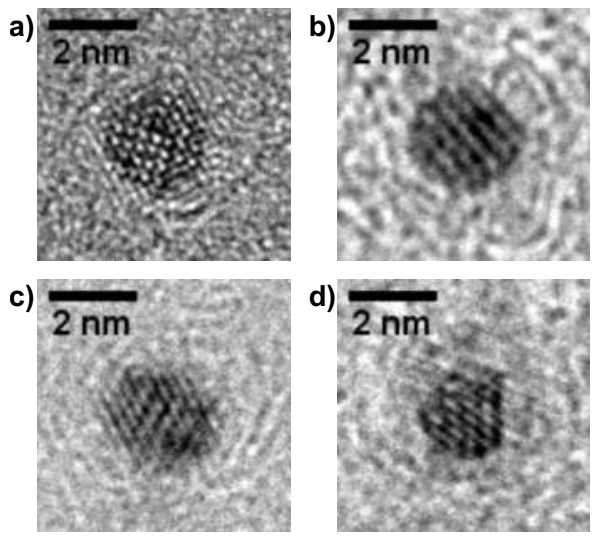

FIG. 4. Small annealed CoPt clusters, around $2 \mathrm{~nm}$ in diameter, showing a $L 1_{0}$ chemical order contrast: (110) in the case of image (a) and (001) for images (b,c,d). Samples corresponding to images (c) and (d) are capped with an a-C layer. even questions the existence, of a critical particle size where chemical order would become unstable at room temperature.

\section{MAGNETIC PROPERTIES}

\section{A. XMCD measurements}

XMCD measurements were performed at the UE56/2PGM-2 beamline of the BESSY synchrotron (Berlin), at the Co $L_{2,3}$ edges. We used a collinear geometry where the applied magnetic field is parallel to the incident photon beam. The x-ray absorption spectroscopy (XAS) signal was monitored using the total electron yield detection mode, with an applied field of $5 \mathrm{~T}$ (which is high enough to fully saturate the sample magnetization, as it has been verified by acquiring hysteresis loops). Measurements were performed at 300 $\mathrm{K}$, on both as-prepared and annealed $\left(2 \mathrm{~h}\right.$ at $650{ }^{\circ} \mathrm{C}$, as for TEM samples) "mille-feuille" samples.

As it can be seen from the XAS spectra (Fig. 5), the a-C matrix appears as an efficient protection against oxidation and corresponds to a low x-ray attenuation, which ensures a high signal-to-noise ratio: The Co $L_{3}$ edge is sharp and does not include any detectable oxide contribution. ${ }^{26}$ Moreover, there is no significant difference in the edges profiles between as-prepared and annealed CoPt particles. Hence, a drastic modification of the Co chemical environment (as carbon diffusion in the clusters) is very unlikely.

The mean orbital and spin magnetic moments per Co atom, $\mu_{L}$ and $\mu_{S}$, have been determined using the wellknown sum rules. ${ }^{59,60}$ Note that, since the samples are made of randomly oriented crystallized nanoparticles, the magnetic dipole term $\mu_{T}$, which reflects the asphericity of the spin moment distribution around the absorbing Co atom, averages to zero. ${ }^{61}$ Therefore, the effective spin moment $\mu_{S}^{\text {eff }}=\mu_{S}+7 \mu_{T}$, evaluated from the XMCD signal, is in fact the true spin magnetic moment.

This assertion can still be moderated, taking into account the fact that the $\mu_{T}$ cancellation may not apply in materials with a strong spin-orbit coupling: In such a case, the dipole term does not only result from the crystal field and consequently cannot be eliminated using angle-dependent XMCD measurements ${ }^{61,62}$ (or, as in the present case, with randomly oriented particles). Moreover, even in materials with a negligible bulk spin-orbit coupling, going to low-dimensional 


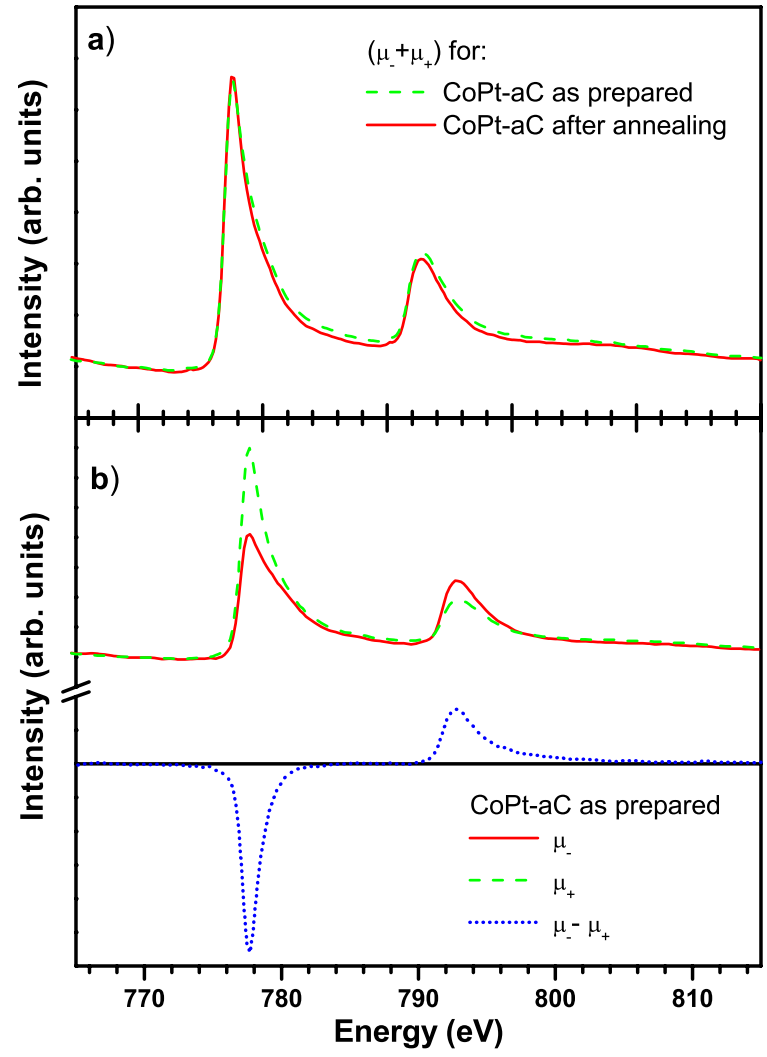

FIG. 5. (Color online) (a) XAS spectra at the Co $L_{3}$ and $L_{2}$ edges for the as-prepared and annealed samples. (b) XAS signals ( $\mu_{-}$and $\mu_{+}$corresponding to the two different circular polarizations) for the as-prepared sample, and the corresponding XMCD curve $\left(\mu_{-}-\mu_{+}\right)$.

systems can result in a failure of the $\mu_{T}$ cancellation. ${ }^{62}$ The reason is that spin-orbit coupling effects are enhanced for low-coordinated atoms. As a matter of fact, even the strict application of the sum rules can be questioned in peculiar situations $^{63}$ (interfaces, low-dimensional systems, etc.). However, contrary to the Fe case, it has been show that both the $\mu_{T}$ cancellation and the sum rules remains verified in the case of low-dimensional Co systems. ${ }^{64,65}$ Anyway, following previously presented studies ${ }^{58,66,67}$ [even for the questionable case of FePt (Refs. 68-70)], we will assume that the sum rules can safely be applied in the case of $\mathrm{CoPt}$ nanoparticles.

The Co magnetic moments, deduced from XMCD, are reported in Table I. There is a striking change of the spin magnetic moment upon annealing: the $\mu_{S}$ value is enhanced by $12 \%$. In addition, both the orbital magnetic moment and the $\mu_{L} / \mu_{S}$ ratio increase significantly. The high $\mu_{S}$ value of the as-prepared sample is consistent with the fact that every Co atom is magnetically ordered, i.e., that there is no dead layer at the clusters surface. To all appearances, the average magnetic moment per $\mathrm{Co}$ atom corresponds to the real $\mathrm{Co}$ moment in CoPt: It is not the result of a balance between some populations of Co atoms with different magnetic moments. The magnetic moment augmentation upon annealing must then be related to an intraparticle change. ${ }^{71}$ According to TEM observations, we claim that this striking evolution is a signature of the structural transition from the chemically disordered to the $L 1_{0}$ phase in CoPt nanoparticles. Amazingly, the net Co magnetic moment is increasing upon chemical ordering, which seems in contradiction with the bulk behavior where the magnetization is decreasing when going from $A 1$ to $L 1_{0}$ phase. ${ }^{32}$ However, this effect could be due to the small size of the particles, or an extensive Pt magnetic moment reduction could compensate the Co magnetic moment variation. Note also that a change in the number of $d$ holes per Co atom, upon annealing, can result in a modification of the Co magnetic moment, as deduced from XMCD. However, since there is also a significant evolution of the $\mu_{L} / \mu_{S}$ ratio, a variation of the number of $d$ holes cannot explain by itself the observed results. In any case, even if such a variation can play a role,$^{72}$ the conclusion remains the same: Annealing has a significant effect on the XMCD signal of CoPt nanoparticles.

Surprisingly, our result significantly differs from the one reported by Grange et al. ${ }^{58}$ In their study, they have determined the Co and Pt magnetic moments of $L 1_{0} \mathrm{CoPt}$ thin films, using XMCD. Assuming their CoPt film is fully chemically ordered (which, according to the authors, is not guaranteed), the discrepancy between the reported value and the present one implies a tremendous size effect. Indeed, such a magnetic moment enhancement is known to happen only at much lower size: The deviation from the bulk behavior occurs around 400 atoms ${ }^{73}$ whereas in our case, the mean CoPt cluster size corresponds to 800 atoms.

Interestingly, the magnetic moment values measured for as-prepared CoPt nanoparticles are very close to those obtained by Grange et al. for a presumably $L 1_{0}$ thin film. A possible interpretation is that chemical order is in fact similar for both CoPt samples: Chemical order in as-prepared $\mathrm{CoPt}$ clusters may be higher than expected (partial order, due to a finite size effect, as discussed by Rohart et al..$^{7}$ ) and/or chemical order in the CoPt thin film may be much lower than

TABLE I. Geometric and magnetic size parameters $\left(d_{m}\right.$ and $\left.w\right)$ deduced, respectively, from TEM and from SQUID measurements; Co spin and orbital magnetic moments $\left[\mu_{S}\right.$ and $\mu_{L}$ are calculated, following Grange et al., assuming that the number of holes per Co atom is $n_{h}=2.628$ (Ref. 58)]; CoPt clusters MAEs $\left(K_{\text {eff }}\right)$ and maximum susceptibility temperatures $\left(T_{\max }\right)$ determined from ZFC curves.

\begin{tabular}{|c|c|c|c|c|c|c|c|c|c|}
\hline & $\begin{array}{c}\text { TEM } \\
d_{m}(\mathrm{~nm})\end{array}$ & $\begin{array}{c}\text { TEM } \\
w\end{array}$ & $\begin{array}{l}\text { SQUID } \\
d_{m}(\mathrm{~nm})\end{array}$ & $\begin{array}{c}\text { SQUID } \\
w\end{array}$ & $\begin{array}{c}\mu_{S} \\
\left(\mu_{B} / \text { at. }\right)\end{array}$ & $\begin{array}{c}\mu_{L} \\
\left(\mu_{B} / \text { at. }\right)\end{array}$ & $\mu_{L} / \mu_{S}$ & $\begin{array}{c}K_{\text {eff }} \\
\left(\mathrm{kJ} / \mathrm{m}^{3}\right)\end{array}$ & $\begin{array}{c}T_{\max } \\
(\mathrm{K})\end{array}$ \\
\hline CoPt as-prepared & $2.60 \pm 0.1$ & $0.42 \pm 0.05$ & $2.55 \pm 0.2$ & $0.36 \pm 0.1$ & 1.70 & 0.12 & 0.071 & $193 \pm 20$ & 28 \\
\hline CoPt annealed & $2.63 \pm 0.1$ & $0.45 \pm 0.05$ & $2.45 \pm 0.2$ & $0.35 \pm 0.1$ & $\begin{array}{c}1.91 \\
(1.76)^{\mathrm{a}}\end{array}$ & $\begin{array}{c}0.18 \\
(0.11)^{\mathrm{a}}\end{array}$ & $\begin{array}{c}0.094 \\
(0.063)^{\mathrm{a}}\end{array}$ & $385 \pm 20$ & 52 \\
\hline
\end{tabular}

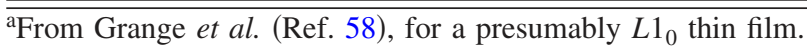


expected. As a consequence, if as-prepared clusters are already partially chemically ordered (however, TEM observations do not substantiate any sizeable chemical order in asprepared particles), it means that the $A 1 \rightarrow L 1_{0}$ transition must have an even higher impact on the Co magnetic moment.

Unfortunately, there is, to our knowledge, no reported value of the Co magnetic moments for the bulk CoPt $A 1$ phase that could have been compared to the present results. On the other hand, Imperia et al. ${ }^{67}$ have recently determined the $\mu_{L} / \mu_{S}$ ratio for disordered Co-Pt alloy nanoparticles. They have found that $\mu_{L} / \mu_{S}$ is significantly higher in their case than in ours. However, due to missing experimental details and characterization, a direct comparison with our system is difficult: The particle size is unknown (the question of an eventual coalescence during sample growth is not evoked), the particles structure and composition are not described, and the use of an oxide capping layer may result in a partial Co oxidation. This oxidation can give rise to a $\mu_{L} / \mu_{S}$ enhancement, as previously demonstrated. ${ }^{75}$ Moreover, we have also observed that CoPt embedded in an oxide matrix $(\mathrm{MgO})$ exhibit a higher $\mu_{L} / \mu_{S}$ ratio than in a-C, ${ }^{26}$ which is consistent with the very high interface magnetic anisotropy measured in $\mathrm{MgO}$ matrix ${ }^{28}$ (as it is expected that a high $\mu_{L}$ goes with a high anisotropy ${ }^{76,77}$ ). Since we are more interested in the intrinsic $\mathrm{CoPt}$ nanoparticles magnetic properties, the choice of an a-C matrix seems to be more judicious than an oxide one.

$\mathrm{XMCD}$ measurements have allowed us to detect a Co orbital moment augmentation, which lets us think of a concomitant magnetic anisotropy increase. However, the magnitude of the $\mu_{L}$ evolution upon annealing $(\simeq 50 \%)$ sheds doubt on the possibility to reach a magnetic anisotropy enhancement as high as in the bulk, where a $\simeq 10000 \%$ augmentation is observed. ${ }^{32}$ Nevertheless, additional experiments are needed in order to obtain a quantitative evaluation of CoPt nanoparticles magnetic anisotropy energy (MAE).

\section{B. SQUID magnetometry measurements}

Magnetic measurements at various temperatures have been performed on the "mille-feuille" samples, using a SQUID magnetometer. The high temperature (300 K) hysteresis loops (see Fig. 6), for as-prepared and annealed samples, do not show any coercivity, which is typical of an assembly of superparamagnetic particles. The magnetization curves can be successfully fitted using a simple Langevin function, taking into account the clusters size distribution and the Co magnetic moment deduced from XMCD results. ${ }^{78}$ This allows us to check that the clusters magnetic size is the same (within error bars) as the geometric size observed by TEM, even for the annealed sample (see Table I). This is another evidence that no coalescence is taking place upon annealing. Moreover, the fact that the magnetic size dispersion is very similar to the geometrical one, shows that the clusters composition distribution must be quite sharp: Indeed, a significant stoichiometry variation among the particles would result in a broadened distribution of magnetic moment per cluster. Besides, since the same Langevin fit can be used for $300 \mathrm{~K}$
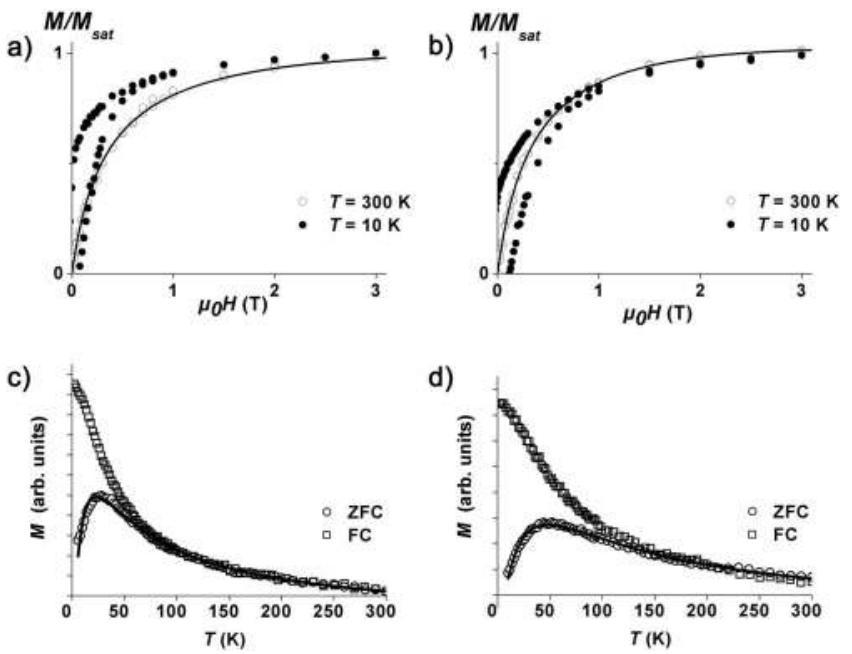

FIG. 6. Hysteresis loops of the as-prepared (a) and annealed (b) sample, at $300 \mathrm{~K}(\bigcirc)$ and $10 \mathrm{~K}(\bullet)$. The solid line corresponds to a fit using a Langevin function, with a log-normal clusters size distribution (parameters given in Table I). ZFC-FC magnetization curves of the as-prepared (c) and annealed (d) sample. The solid line corresponds to a fit using a theoretical semianalytical model (see text), with the same clusters size distribution as for the high temperature cycles fit.

and $200 \mathrm{~K}$ hysteresis cycles, it means that anisotropy effects are negligible at such temperatures.

Oppositely, magnetization cycles at low temperature (10 $\mathrm{K})$ exhibit a coercive field and a remanent to saturation magnetization ratio $M_{r} / M_{\text {sat }} \simeq 0.4$ (see Fig. 6). Note that the maximum magnetic field $\left(\mu_{0} H=5 \mathrm{~T}\right)$ is largely sufficient to saturate the magnetization. According to the StonerWohlfarth model, ${ }^{79} M_{r} / M_{\text {sat }}$ must be lower than 0.5 for an assembly of randomly oriented macrospins without interaction. This gives an indication that the interparticle distance in the samples is large enough to consider that the CoPt clusters are isolated.

The effect of annealing (i.e., chemical ordering) is immediately visible on the low temperature cycle: The coercive field $\mu_{0} H_{C}$ increases twofold (from $0.07 \mathrm{~T}$ to $0.12 \mathrm{~T}$ ), meaning that the magnetic anisotropy is enhanced. In order to get a quantitative estimation of the MAE, we have performed magnetic susceptibility measurements following the zerofield-cooled and/or field-cooled (ZFC-FC) procedure. We use a 50 Oe (i.e., $0.005 \mathrm{~T}$ ) magnetic field, applied in a direction parallel to the sample plane. As it can be seen in Fig. 6, the ZFC curves show a transition from ferromagnetic to superparamagnetic regime, as evidenced by the susceptibility peak around a given $T_{\max }$ temperature. The $1 / T$ behavior, for high enough temperature ( $T$ slightly larger than $T_{\max }$ ) is consistent with the picture of rapidly switching macrospins without interaction. Just as for $H_{C}$, the $T_{\max }$ value is doubled upon annealing, which is another signature of a strong MAE augmentation.

The simple relation $K_{\text {eff }} V=25 k_{B} T_{\text {max }}$ is used in many studies to derive the anisotropy constant $K_{\text {eff }}$ (MAE) from the measured value of $T_{\max }$ and knowing the typical particles volume $V$. However, the application of this "rule of thumb" equation can be highly uncertain: The particles size disper- 
sion can only be approximately taken into account (and is not considered in most cases); the use of $T_{\max }$ instead of the blocking temperature (which is hard to define for particle distributions) is an approximation; and the value of the prefactor (usually 25) is not well established and varies among the authors.

Nevertheless, this crude expression can be used to get a first MAE estimation and gives in the present case $K_{\text {eff }}$ $=0.25 \mathrm{MJ} / \mathrm{m}^{3}\left(0.25 \times 10^{7} \mathrm{erg} / \mathrm{cm}^{3}\right)$ and $K_{\text {eff }}=0.50 \mathrm{MJ} / \mathrm{m}^{3}$ $\left(0.50 \times 10^{7} \mathrm{erg} / \mathrm{cm}^{3}\right)$, respectively, for the chemically disordered and chemically ordered CoPt nanoparticles. Following Dormann et al. ${ }^{80}$ (as Petit et al. $\mathrm{did}^{81}$ ), we have used the effective volume $V_{\text {eff }}=\left\langle V^{2}\right\rangle /\langle V\rangle$ in the equation.

A more elaborate approach is yet possible, where the entire ZFC curve is adjusted by a semianalytical model (described in Ref. 28), which fully takes into account the particles size distribution and the dynamic temperature sweep during the experimental acquisition. The only adjustable parameter is then the anisotropy constant $K_{\text {eff: }}$ The corresponding energy barrier of a cluster with volume $V$ is simply written as $\Delta E=K_{\text {eff }} V$. This widely used expression implicitly supposes that the clusters size distribution is the major source of dispersion for the magnetization switching energy barrier. As a matter of fact, this assumption is not firmly justified, especially in the case of an alloy: In addition to the usual surface and shape distribution effects (additional facets on a perfect truncated octahedron for instance ${ }^{82}$, compositional variation and chemical order distribution ${ }^{83,84}$ (or even, as discussed by Rohart et al.,${ }^{74}$ the statistical atomic distribution at a fixed chemical order parameter) can deeply modify the MAE. Moreover, it is the standard procedure to also neglect any temperature variation of the MAE and of the cluster magnetization, which is quite justified in the temperature range considered here. ${ }^{85,86}$ Anyway, since the simple relation $\Delta E=K_{\text {eff }} V$ appears to be satisfactory in the present case, we will not go further into a cluster anisotropy analysis in this study. An additional degree of freedom, using a surface anisotropy term (i.e., $\Delta E=K_{V} V+K_{S} S$, where $S$ is the cluster surface), does not change the results and just leads to $K_{S} \simeq 0$.

This semianalytical model has been applied to adjust the ZFC curves, using a log-normal function for the clusters magnetic size distribution, with the parameters $d_{m}$ and $w$ [see Eq. (1)] determined from the Langevin fit of high temperature hysteresis loops. We have been able to precisely reproduce the experimental curves (see Fig. 6), the best fit giving a MAE value of $K_{\text {eff }}=0.19 \mathrm{MJ} / \mathrm{m}^{3}\left(0.19 \times 10^{7} \mathrm{erg} / \mathrm{cm}^{3}\right)$ for chemically disordered $\mathrm{CoPt}$ nanoparticles and $K_{\text {eff }}$ $=0.38 \mathrm{MJ} / \mathrm{m}^{3} \quad\left(0.38 \times 10^{7} \mathrm{erg} / \mathrm{cm}^{3}\right)$ for $L 1_{0}$ ones. The $100 \%$ MAE relative variation found with this model is consistent with the $T_{\max }$ doubling, if we simply consider the "rule of thumb" relation previously mentioned (since, in the present case, we know that the clusters size distribution is the same for as-prepared and annealed samples).

\section{DISCUSSION}

The TEM observations, together with the magnetic measurements previously presented show that the CoPt nanopar- ticles evolve upon annealing: The structure changes from $A 1$ to $L 1_{0}$; the Co magnetic moment is enhanced as well as the $\mu_{L} / \mu_{S}$ ratio; and the MAE increases (consequently, the blocking temperature is higher). Let us emphasize that the particles embedded in a-C remain almost isolated with no significant change in size, so that the magnetic properties evolution really reflects the behavior of individual $\mathrm{CoPt}$ nanoparticles. Surprisingly, although the anisotropy is found to increase with chemical ordering, the determined MAE value is quite small as compared to the bulk. ${ }^{32,33}$ The magnitude of the corresponding MAE variation is significant but dramatically lower than the two orders of magnitude augmentation reported for the bulk!

In order to fix the ideas, the blocking temperature of a 3 $\mathrm{nm}$ particle with the same MAE as the bulk CoPt $L 1_{0}$ one $\left(5 \mathrm{MJ} / \mathrm{m}^{3}\right)$ would be $\simeq 200 \mathrm{~K}$. Conversely, the particle size that would account for the observed blocking temperature $(52 \mathrm{~K})$, if the MAE was the bulk one, would be $1.9 \mathrm{~nm}$ in diameter.

Our unexpected results can be compared to previous magnetic measurements carried on $\mathrm{CoPt}$ nanoparticles. ${ }^{16-24,27-30,81,87}$ Many researchers have reported extremely high coercivity for annealed CoPt samples and some have derived MAE values. ${ }^{21,81,87}$ However, to our knowledge, among the previously presented results, there is not any case where the system was made of noninteracting particles (as it can be seen from the remanence to saturation ratio $M_{r} / M_{\text {sat }}$ which, according to the isolated macrospin model,${ }^{79}$ should be 0.5 at $0 \mathrm{~K}$ and then decrease with temperature). In most cases, the impact of annealing on the particles size (in particular, coalescence) has not been investigated. Moreover, very often, there is no definite proof that the system is made of chemically ordered CoPt nanoparticles, so that the evolution of the magnetic properties, which is ascribed to $L 1_{0}$ chemical ordering, could come (at least, partly) from particle growth and interparticle interactions. It must also be noted that the CoPt particles studied in the present case are among the smallest ever reported. Here, contrary to other works, several characterizations have been made on the very same system (and even on the same sample for XMCD and SQUID measurements). Therefore, our conclusions must be particularly reliable.

One can envisage various effects to account for the striking difference between the MAE of the bulk $L 1_{0}$ phase and the one of annealed CoPt nanoparticles. Indeed, one may think that (i) the clusters magnetic size is smaller than expected; (ii) there are interparticle interactions; (iii) the particles are not fully chemically ordered; (iv) the MAE reduction is a finite size effect; (v) the MAE reduction is due to the a-C matrix. We will discuss these different points in the following.

(i) The magnetic size could indeed be different (smaller in fact), than the diameter observed by TEM. However, as said before, to be compatible with the bulk $L 1_{0}$ MAE, the mean magnetic diameter should be around $1.9 \mathrm{~nm}$. This would imply that the major part of Co atoms are in fact nonmagnetic ("dead-layer" effect), which is not compatible with XMCD observations. Moreover, as presented in the section reporting SQUID results, the size distribution determined from TEM is able to reproduce the high temperature superparamagnetic 
magnetization cycles. Clusters with a much smaller magnetic size would definitely not produce a correct magnetization curve. This explanation can then be rejected.

(ii) Magnetic interactions between particles can modify the blocking temperature and change the magnetization curves. ${ }^{88-90}$ However, for blocked particles, interactions would broaden the switching field distribution and shift it toward higher values ${ }^{91}$ (as if the particles volume was greater, thus increasing the energy barrier for magnetization reversal): If there were some interactions between particles, the presently measured MAE would be overestimated meaning that the true MAE reduction, as compared to the bulk, is in fact even higher. Consequently, the possibility of nonvanishing interparticle interactions cannot account for the observed effect.

(iii) The $L 1_{0}$ chemical order could be incomplete and/or some particles (the smallest ones) may still be in the $A 1$ phase. However, if it is the case, it means that the Co magnetic moment enhancement, which is an average value over all Co atoms, is even greater for a complete $A 1 \rightarrow L 1_{0}$ transition. Moreover, since a $L 1_{0}$-type contrast is highly visible by HRTEM and since a perfectly ordered nanocrystal is in very good agreement with experimental images (see Fig. 3), the chemical order parameter must be significant. ${ }^{92}$ In addition, we can safely assume that a $2 \mathrm{~h}$ annealing at $650{ }^{\circ} \mathrm{C}$ is enough to reach thermodynamic equilibrium, so that there is no reason for identical particles to be in different phases. Nevertheless, it cannot be excluded that, because of the size dependence of the transition temperature, the smallest particles in the sample remain chemically disordered.

Following this idea, we have tried to determine a critical diameter under which the particles would remain in the $A 1$ phase, by fitting the ZFC curve with the bulk MAE value for $L 1_{0}$ clusters (largest particles) and the previously determined MAE for $A 1$ clusters (smallest particles). We have found that it is definitely impossible to account for the experimental observations with a part of the clusters size distribution corresponding to particles having the bulk $L 1_{0}$ MAE. Conversely, assuming that the critical diameter is $2 \mathrm{~nm}$ (chemically ordered particles of this size have been observed by TEM), we have determined the MAE of the largest clusters, which are in the $L 1_{0}$ phase: It does not change, within the uncertainty, the MAE value previously determined by assuming that all the particles are in the ordered phase. In the end, even if some particles in the annealed sample are still partially disordered, it does not change the conclusion that the MAE of $L 1_{0}$ CoPt nanoparticle is much lower than the bulk one.

(iv) The finite size of the CoPt clusters, and the corresponding high surface to volume ratio could be responsible for the striking MAE reduction observed in the case of $L 1_{0}$ nanoparticles. Actually, Rohart et al. ${ }^{74}$ recently predicted, using an empirical Néel anisotropy model, that the MAE of $L 1_{0}$ particles should decrease when going to small clusters. This counterintuitive result can be written in term of a finite size correction to the anisotropy: $K_{\text {eff }}(N)=K_{\infty}+K_{\mathrm{fs}} / N^{-\alpha}$, where $N$ is the number of atoms in the cluster, and $K_{\infty}$ and $K_{\mathrm{fs}}$ are, respectively, the bulk MAE and the finite size contribution to the MAE. These two terms, together with the $\alpha$ exponent, vary with the cluster chemical order parameter. The Néel model predicts that $K_{\mathrm{fs}}$ is negative for CoPt clusters with a significant chemical order parameter (and in particular for perfect $L 1_{0}$ particles), while it is positive for chemically disordered clusters. This is qualitatively consistent with our results where as-prepared particles display a higher $\mathrm{MAE}^{93}$ than the bulk (according to Ref. 32, the bulk MAE of the CoPt $A 1$ phase is around $0.06 \mathrm{MJ} / \mathrm{m}^{3}$ ), whereas it is the opposite for chemically ordered nanoparticles.

However, the magnitude of the calculated finite size effect is not in agreement with the MAE reduction observed in the present case. This may be due to other contributions neglected in the crude Néel theoretical model. In particular, magnetoelastic effects could dominate and have strong repercussions on the size dependence of the MAE. For instance, the crystallographic structure of CoPt nanoparticles can be slightly different from the bulk one, the tetragonalization can differ from the one observed in the bulk, resulting in modified nearest-neighbor distances. A consequence could be a significative lowering of the magnetic anisotropy. Even so, such a weak structural distortion is hard to detect, and its precise impact on the magnetic properties is even more difficult to determine, so that such a magnetoelastic effect is quite speculative. Another possibility is that some particles may not be made of a single $L 1_{0}$ domain: Twinned clusters for instance could correspond to particles with various $c$ axes (easy magnetization direction), leading to a strong MAE diminution. ${ }^{7}$ Nevertheless, if some twinned (and multiply twinned) particles have indeed been detected by TEM, the observation of such structures remains exceptional, especially in the annealed sample. These considerations show how size reduction effects could play a major role in the MAE decrease of CoPt $L 1_{0}$ nanoparticles. However, it also implies that a complete analysis should explicitly consider that the MAE is in fact size dependent. ${ }^{94}$

(v) The last possibility is that the a-C matrix ${ }^{95}$ strongly modifies the clusters magnetic properties and in particular the magnetic anisotropy. Even if such a hypothesis cannot be firmly discarded, some elements tend to show that carbon does not have such an impact on CoPt nanoparticles. First, as mentioned earlier, no alloying effect (carbide formation) is detected from the core level absorption peak of Co. This is also consistent with the fact that, at the macroscopic scale, the formation of cobalt and platinum carbide is not favorable. Second, the use of a carbon matrix has no detectable effect on the structure of CoPt clusters, even after annealing: $L 1_{0}$ clusters are observed both with and without the a-C matrix. Third, as demonstrated by the high Co magnetic moment value measured by XMCD, carbon does not quench cobalt magnetism (contrary to other elements, oxygen in particular). Moreover, if the carbon matrix induces a pure interface effect, we expect that it could be accounted for with a surface anisotropy term in the ZFC curves fit. Since it is not the case, a supposed magnetic effect of carbon should have repercussions on the volume magnetic anisotropy. It is still possible to envisage carbon diffusion in the CoPt clusters core, resulting in interstitial carbon atoms with a dramatic effect on the magnetic anisotropy, but such an unprecedented phenomenon remains highly speculative.

The true explanation of the observed reduced magnetic anisotropy of $\mathrm{CoPt} L 1_{0}$ clusters, as compared to the bulk, 
may be a combination of the various envisaged effects. Identifying these physical mechanisms, and trying to control them, is a prerequisite to be able to overcome the intrinsic, and unexpected, limitations on the magnetic anisotropy of CoPt nanoparticles.

\section{CONCLUSION}

In conclusion, we have studied the structural and magnetic properties of $\mathrm{CoPt}$ nanoparticles synthesized by the LECBD technique, in ultrahigh vacuum conditions. The produced samples are made of nearly isolated nanoparticles embedded in amorphous carbon. The $A 1 \rightarrow L 1_{0}$ phase transformation takes place upon annealing, without particle coalescence, as evidence by TEM. A chemical contrast revealing the $L 1_{0}$ order has been observed by HRTEM, even for CoPt particles as small as $2 \mathrm{~nm}$ in diameter. XMCD measurements have shown that chemical ordering is accompanied by a striking enhancement of the Co magnetic moment. A 100\% magnetic anisotropy increase has also been evidenced by SQUID magnetometry measurements on the very same samples. However, the MAE of annealed clusters, for which the $L 1_{0}$ chemical order has been evidenced, ap- pears to be much lower than the bulk one (by more than one order of magnitude).

A careful examination of the previously presented results reveals that the present study is the first intrinsic magnetic characterization of well-known $\mathrm{CoPt}$ nanoparticles. This means that the stimulating results reported for CoPt particles samples may in fact have been over interpreted. Therefore, one can legitimately question the ability of CoPt to keep its promises: The intrinsic magnetic anisotropy of chemically ordered CoPt nanoparticles may never be high enough to ensure a magnetization thermal stability compatible with practical applications. Let us note that the same problem could be met for FePt nanoparticles, where the even more numerous magnetic characterizations reported should be cautiously examined.

\section{ACKNOWLEDGMENTS}

The authors gratefully acknowledge E. Bernstein, A. Perez, O. Boisron, and G. Guiraud for stimulating discussions and for their support. The authors acknowledge support through the BESSY IA-SFS program (Contracts No. BESSYID.07.1.672 and No. RII 3CT-2004-506008). *ftournus@1pmcn.univ-lyon1.fr

${ }^{1}$ I. M. L. Billas, A. Chatelain, and W. A. de Heer, Science 265, 1682 (1994).

${ }^{2}$ P. Gambardella, S. Rusponi, M. Veronese, S. S. Dhesi, C. Grazioli, A. Dallmeyer, I. Cabria, R. Zeller, P. H. Dederichs, K. Kern, C. Carbone, and H. Brune, Science 300, 1130 (2003).

${ }^{3}$ D. J. Sellmyer, M. Yu, and R. D. Kirby, Nanostruct. Mater. 12, 1021 (1999).

${ }^{4}$ S. H. Sun, C. B. Murray, D. Weller, L. Folks, and A. Moser, Science 287, 1989 (2000).

${ }^{5}$ S. H. Sun, S. Anders, T. Thomson, J. E. E. Baglin, M. F. Toney, H. F. Hamann, C. B. Murray, and B. D. Terris, J. Phys. Chem. B 107, 5419 (2003).

${ }^{6}$ S. Stoyanov, Y. Huang, Y. Zhang, V. Skumryev, G. C. Hadjipanayis, and D. Weller, J. Appl. Phys. 93, 7190 (2003).

${ }^{7}$ B. Rellinghaus, S. Stappert, M. Acet, and E. F. Wassermann, J. Magn. Magn. Mater. 266, 142 (2003).

${ }^{8}$ B. Stahl, J. Ellrich, R. Theissmann, M. Ghafari, S. Bhattacharya, H. Hahn, N. S. Gajbhiye, D. Kramer, R. N. Viswanath, J. Weissmüller, and H. Gleiter, Phys. Rev. B 67, 014422 (2003).

${ }^{9}$ S. Stappert, B. Rellinghaus, M. Acet, and E. F. Wassermann, J. Cryst. Growth 252, 440 (2003).

${ }^{10}$ H.-G. Boyen, K. Fauth, B. Stahl, P. Ziemann, G. Kästle, F. Weigl, F. Banhart, M. Hessler, G. Schütz, N. Gajbhiye, J. Ellrich, H. Hahn, M. Büttner, M. G. Garnier, and P. Oelhafen, Adv. Mater. 17, 574 (2005).

${ }^{11}$ K. Elkins, D. Li, N. Poudyal, V. Nandwana, Z. Jin, K. Chen, and J. P. Liu, J. Phys. D 38, 2306 (2005).

${ }^{12}$ M. Takahashi, T. Ogawa, D. Hasegawa, and B. Jeyadevan, J. Appl. Phys. 97, 10 J307 (2005).

${ }^{13}$ H. Nguyen, L. Howard, G. Stinton, S. Giblin, B. Tanner, I. Terry, A. Hughes, I. Ross, A. Serres, and J. Evans, Chem. Mater. 18,
6414 (2006).

${ }^{14}$ V. Nandwana, K. E. Elkins, N. Poudyal, G. S. Chaubey, K. Yano, and J. P. Liu, J. Phys. Chem. C 111, 4185 (2007).

${ }^{15}$ Chuan-bing Rong, N. Poudyal, G. S. Chaubey, V. Nandwana, R. Skomski, Y. Q. Wu, M. J. Kramer, and J. P. Liu, J. Appl. Phys. 102, 043913 (2007).

${ }^{16}$ M. Yu, Y. Liu, and D. J. Sellmyer, J. Appl. Phys. 87, 6959 (2000).

${ }^{17}$ A. C. C. Yu, M. Mizuno, Y. Sasaki, H. Kondo, and K. Hiraga, Appl. Phys. Lett. 81, 3768 (2002).

${ }^{18}$ Y. Xu, Z. G. Sun, Y. Qiang, and D. J. Sellmyer, J. Magn. Magn. Mater. 266, 164 (2003).

${ }^{19}$ C. N. Chinnasamy, B. Jeyadevan, K. Shinoda, and K. Tohji, J. Appl. Phys. 93, 7583 (2003).

${ }^{20}$ Y. Sui, L. Yue, R. Skomski, X. Z. Li, J. Zhou, and D. J. Sellmyer, J. Appl. Phys. 93, 7571 (2003).

${ }^{21}$ X. Sun, Z. Y. Jia, Y. H. Huang, J. W. Harrell, D. E. Nikles, K. Sun, and L. M. Wang, J. Appl. Phys. 95, 6747 (2004).

${ }^{22}$ L. Castaldi, K. Giannakopoulos, A. Travlos, D. Niarchos, S. Boukari, and E. Beaurepaire, J. Magn. Magn. Mater. 290-291, 544 (2005).

${ }^{23}$ M. Mizuno et al., J. Appl. Phys. 97, 10J301 (2005).

${ }^{24}$ J.-M. Qiu, Y.-H. Xu, J. H. Judy, and J.-P. Wang, J. Appl. Phys. 97, 10 P704 (2005).

${ }^{25}$ A. Hannour, L. Bardotti, B. Prevel, E. Bernstein, P. Melinon, A. Perez, J. Gierak, E. Bourhis, and D. Mailly, Surf. Sci. 594, 1 (2005).

${ }^{26}$ L. Favre et al., Phys. Rev. B 74, 014439 (2006).

${ }^{27}$ T. Seto, K. Koga, H. Akinaga, F. Takano, T. Orii, and M. Hirasawa, J. Nanopart. Res. 8, 371 (2006).

${ }^{28}$ S. Rohart, C. Raufast, L. Favre, E. Bernstein, E. Bonet, and V. Dupuis, Phys. Rev. B 74, 104408 (2006). 
${ }^{29}$ J. H. Kim, J. Kim, N. Oh, Y.-H. Kim, C. K. Kim, C. S. Yoon, and S. Jin, Appl. Phys. Lett. 90, 023117 (2007).

${ }^{30}$ D. M. Newman, M. L. Wears, M. Jollie, and D. Choo, Nanotechnology 18, 205301 (2007).

${ }^{31}$ D. Alloyeau, C. Langlois, C. Ricolleau, Y. L. Bouar, and A. Loiseau, Nanotechnology 18, 375301 (2007).

${ }^{32}$ R. A. McCurrie and P. Gaunt, Philos. Mag. 13, 567 (1966).

${ }^{33}$ G. Hadjipanayis and P. Gaunt, J. Appl. Phys. 50, 2358 (1979).

${ }^{34}$ Y. K. Takahashi, T. Ohkubo, M. Ohnuma, and K. Hono, J. Appl. Phys. 93, 7166 (2003).

${ }^{35}$ Y. K. Takahashi, T. Koyama, M. Ohnuma, T. Ohkubo, and K. Hono, J. Appl. Phys. 95, 2690 (2004).

${ }^{36}$ S. Fukami and N. Tanaka, Philos. Mag. Lett. 84, 33 (2004).

${ }^{37}$ T. Miyazaki, O. Kitakami, S. Okamoto, Y. Shimada, Z. Akase, Y. Murakami, D. Shindo, Y. K. Takahashi, and K. Hono, Phys. Rev. B 72, 144419 (2005).

${ }^{38}$ R. V. Chepulskii and W. H. Butler, Phys. Rev. B 72, 134205 (2005).

${ }^{39}$ B. Yang, M. Asta, O. N. Mryasov, T. J. Klemmer, and R. W. Chantrell, Scr. Mater. 53, 417 (2005).

${ }^{40}$ B. Yang, M. Asta, O. N. Mryasov, T. J. Klemmer, and R. W. Chantrell, Acta Mater. 54, 4201 (2006).

${ }^{41}$ C.-b. Rong, D. Li, V. Nandwana, N. Poudyal, Y. Ding, Z. Wang, H. Zeng, and J. Liu, Adv. Mater. 18, 2984 (2006).

${ }^{42}$ M. Muller, P. Erhart, and K. Albe, Phys. Rev. B 76, 155412 (2007).

${ }^{43}$ Z. Dai, S. Sun, and Z. Wang, Nano Lett. 1, 443 (2001).

${ }^{44}$ T. Thomson, S. L. Lee, M. F. Toney, C. D. Dewhurst, F. Y. Ogrin, C. J. Oates, and S. Sun, Phys. Rev. B 72, 064441 (2005).

${ }^{45}$ B. Yao, R. V. Petrova, R. R. Vanfleet, and K. R. Coffey, J. Appl. Phys. 99, 08E913 (2006).

${ }^{46}$ H. Wang, Y. Huang, Y. Zhang, G. Hadjipanayis, D. Weller, and A. Simopoulos, J. Magn. Magn. Mater. 310, 22 (2007).

${ }^{47}$ As discussed further in this paper.

${ }^{48}$ P. Melinon, V. Paillard, V. Dupuis, A. Perez, P. Jensen, A. Hoareau, M. Broyer, J. L. Vialle, M. Pellarin, B. Baguenard, and J. Lerme, Int. J. Mod. Phys. B 9, 339 (1995).

${ }^{49}$ A. Perez, P. Melinon, V. Dupuis, B. Prevel, L. Bardotti, J. Tuaillon-Combes, B. Masenelli, M. Treilleux, M. Pellarin, J. Lerme, E. Cottancin, M. Broyer, M. Jamet, M. Negrier, F. Tournus, and M. Gaudry, Mater. Trans. 42, 1460 (2001).

${ }^{50}$ T. G. Dietz, M. A. Duncan, D. E. Powers, and R. E. Smalley, J. Chem. Phys. 74, 6511 (1981).

${ }^{51}$ P. Milani and W. A. deHeer, Rev. Sci. Instrum. 61, 1835 (1990).

${ }^{52}$ A. Hannour, Ph.D. thesis, Université Lyon 1, 2006.

${ }^{53}$ M. Jamet, W. Wernsdorfer, C. Thirion, D. Mailly, V. Dupuis, P. Mélinon, and A. Pérez, Phys. Rev. Lett. 86, 4676 (2001).

${ }^{54}$ P. Stadelmann, Microsc. Microanal. 9, 60 (2003).

${ }^{55}$ HRTEM multislice calculations are performed using a "sliced" CoPt cluster (slice thickness of $1.902 \AA$ ) on amorphous carbon ( $3 \mathrm{~nm}$ thick), with a supercell of a $9.95 \mathrm{~nm}$ side length. The electron scattering potential and the wave-function transmission are calculated with a $1024 \times 1024$ fast-Fourier-transform (maximum spatial frequency $\simeq 10 \AA^{-1}$ ) corresponding to a pixel size of $\simeq 0.1 \AA$ in the simulated image. For the high-resolution image formation, the microscope parameters are fixed to the value corresponding to the experimentally used electron microscope (JEOL 2010F).

${ }^{56}$ Note that in Ref. 29 there are claims to having observed $L 1_{0}$ CoPt particles by HRTEM, whereas no such contrast was visible on their images, etc.
${ }^{57}$ To our knowledge, models only exist for the very similar FePt system (see Refs. 34-42).

${ }^{58}$ W. Grange, I. Galanakis, M. Alouani, M. Maret, J.-P. Kappler, and A. Rogalev, Phys. Rev. B 62, 1157 (2000).

${ }^{59}$ B. T. Thole, P. Carra, F. Sette, and G. van der Laan, Phys. Rev. Lett. 68, 1943 (1992).

${ }^{60}$ P. Carra, B. T. Thole, M. Altarelli, and X. Wang, Phys. Rev. Lett. 70, 694 (1993).

${ }^{61}$ J. Stöhr, J. Electron Spectrosc. Relat. Phenom. 75, 253 (1995).

${ }^{62}$ C. Ederer, M. Komelj, J. W. Davenport, and M. Fahnle, J. Electron Spectrosc. Relat. Phenom. 130, 97 (2003).

${ }^{63}$ C. Ederer, M. Komelj, M. Fähnle, and G. Schütz, Phys. Rev. B 66, 094413 (2002).

${ }^{64}$ M. Komelj, C. Ederer, J. W. Davenport, and M. Fähnle, Phys. Rev. B 66, 140407(R) (2002).

${ }^{65}$ C. Ederer, M. Komelj, and M. Fähnle, Phys. Rev. B 68, 052402 (2003).

${ }^{66}$ W. Grange, M. Maret, J.-P. Kappler, J. Vogel, A. Fontaine, F. Petroff, G. Krill, A. Rogalev, J. Goulon, M. Finazzi, and N. B. Brookes, Phys. Rev. B 58, 6298 (1998).

${ }^{67}$ P. Imperia, P. Andreazza, D. Schmitz, J. Penuelas, and C. Andreazza-Vignolle, J. Magn. Magn. Mater. 310, 2417 (2007).

${ }^{68}$ C. Antoniak, J. Lindner, M. Spasova, D. Sudfeld, M. Acet, M. Farle, K. Fauth, U. Wiedwald, H.-G. Boyen, P. Ziemann, F. Wilhelm, A. Rogalev, and S. Sun, Phys. Rev. Lett. 97, 117201 (2006).

${ }^{69}$ O. Dmitrieva, M. Spasova, C. Antoniak, M. Acet, G. Dumpich, J. Kastner, M. Farle, K. Fauth, U. Wiedwald, H.-G. Boyen, and P. Ziemann, Phys. Rev. B 76, 064414 (2007).

${ }^{70}$ N. Jaouen, D. Babonneau, J. M. Tonnerre, D. Carbone, F. Wilhelm, A. Rogalev, T. K. Johal, and G. van der Laan, Phys. Rev. B 76, 104421 (2007).

${ }^{71}$ Considering only the XMCD results, one could think of a Co segregation at the clusters surface, which could result in a magnetic moment increase. However, this hypothesis is not compatible with the HRTEM observations: With a Co segregation, the $L 1_{0}$ phase formation in the clusters core would not be possible (problem of composition).

${ }^{72}$ Unfortunately, we are not aware of any experimental or theoretical determination of the evolution of the number of $d$ holes per Co atom, when going from the chemically disordered to the chemically ordered phase of CoPt.

${ }^{73}$ I. M. L. Billas, A. Chatelain, and W. A. de Heer, J. Magn. Magn. Mater. 168, 64 (1997).

${ }^{74}$ S. Rohart, F. Tournus, and V. Dupuis (unpublished).

${ }^{75}$ P. Imperia, D. Schmitz, H. Maletta, N. S. Sobal, and M. Giersig, Phys. Rev. B 72, 014448 (2005).

${ }^{76}$ P. Bruno, Phys. Rev. B 39, 865 (1989).

${ }^{77}$ G. van der Laan, J. Phys.: Condens. Matter 10, 3239 (1998).

${ }^{78}$ For Pt, we use the value reported in Ref. 58.

${ }^{79}$ E. C. Stoner and E. P. Wohlfarth, Philos. Trans. R. Soc. London, Ser. A 240, 599 (1948).

${ }^{80}$ J. L. Dormann, F. D’Orazio, F. Lucari, E. Tronc, P. Prené, J. P. Jolivet, D. Fiorani, R. Cherkaoui, and M. Noguès, Phys. Rev. B 53, 14291 (1996).

${ }^{81}$ C. Petit, S. Rusponi, and H. Brune, J. Appl. Phys. 95, 4251 (2004).

${ }^{82}$ M. Jamet, W. Wernsdorfer, C. Thirion, V. Dupuis, P. Melinon, A. Perez, and D. Mailly, Phys. Rev. B 69, 024401 (2004).

${ }^{83}$ S. S. A. Razee, J. B. Staunton, B. Ginatempo, E. Bruno, and F. J. 
Pinski, Phys. Rev. B 64, 014411 (2001).

${ }^{84}$ J. B. Staunton, S. Ostanin, S. S. A. Razee, B. Gyorffy, L. Szunyogh, B. Ginatempo, and E. Bruno, J. Phys.: Condens. Matter 16, S5623 (2004).

${ }^{85}$ J. B. Staunton, S. Ostanin, S. S. A. Razee, B. L. Gyorffy, L. Szunyogh, B. Ginatempo, and E. Bruno, Phys. Rev. Lett. 93, 257204 (2004).

${ }^{86}$ O. N. Mryasov, U. Nowak, K. Y. Guslienko, and R. W. Chantrell, Europhys. Lett. 69, 805 (2005).

${ }^{87}$ R. K. Rakshit and R. C. Budhani, J. Phys. D 39, 1743 (2006).

${ }^{88}$ R. W. Chantrell, N. Walmsley, J. Gore, and M. Maylin, Phys. Rev. B 63, 024410 (2000).

${ }^{89}$ J. M. Vargas, W. C. Nunes, L. M. Socolovsky, M. Knobel, and D. Zanchet, Phys. Rev. B 72, 184428 (2005).

${ }^{90}$ W. Figueiredo and W. Schwarzacher, J. Phys.: Condens. Matter 19, 276203 (2007).
${ }^{91}$ O. A. Chubykalo and R. W. Chantrell, J. Magn. Magn. Mater. 272-276, E1169 (2004).

${ }^{92}$ We are currently working on a quantitative determination of the chemical order parameter of a single particle from HRTEM images.

${ }^{93}$ The MAE is also higher than some values reported for other as-prepared CoPt particles (see Refs. 81 and 87).

${ }^{94}$ It seriously complicates the MAE determination from ZFC curves fit. Unless the expected size dependence of the MAE is well established, the results will not gain in precision by just adding adjustable parameters. Therefore, we think it is wiser to keep using the simple $\Delta E=K_{\mathrm{eff}} V$ model when it is successful. The best way to directly study the MAE size dependence would be to use samples made of size-selected particles: We are currently working in this direction.

${ }^{95}$ Or some undetected impurities. 